\title{
Survival and prognosticators of gastric cancer that recurs after adjuvant chemotherapy with S-1
}

\author{
Toru Aoyama $\cdot$ Takaki Yoshikawa \\ Takafumi Watanabe · Tsutomu Hayashi • \\ Takashi Ogata $\cdot$ Haruhiko Cho $\cdot$ Akira Tsuburaya
}

Received: 10 August 2010/Accepted: 1 December 2010/Published online: 16 February 2011

(C) The International Gastric Cancer Association and The Japanese Gastric Cancer Association 2011

\begin{abstract}
Background Some patients experience a recurrence of cancer even after curative D2 gastrectomy followed by adjuvant S-1 chemotherapy. The objective of this retrospective study was to clarify the survival and prognosticators in these patients.

Methods The study selected patients who underwent curative D2 surgery, were diagnosed with stage II, IIIA, or IIIB cancer, received adjuvant S-1 for more than 4 weeks, and experienced recurrence confirmed by an imaging study.

Results A total of 34 patients were evaluated. The median overall survival (OS) was significantly longer in the 26 patients who received palliative chemotherapy than that in the 8 who did not ( 8.5 vs. 2.5 months, $P=0.002)$. Only 1 patient received $S-1,21$ received taxane-containing regimens, and 4 received irinotecan plus cisplatin as the firstline chemotherapy. Univariate and multivariate analyses showed that the histological type was only independent significant prognosticator.

Conclusions These results suggested that the survival did not reach the level expected for first-line chemotherapy. The histological type was a significant prognosticator in patients who experienced recurrence after adjuvant S-1 therapy and thereafter received palliative chemotherapy.
\end{abstract}

Keywords Gastric cancer - Adjuvant chemotherapy · Recurrence $\cdot$ S-1

T. Aoyama · T. Yoshikawa $(\varangle) \cdot$ T. Watanabe $\cdot$ T. Hayashi . T. Ogata $\cdot$ H. Cho $\cdot$ A. Tsuburaya

Department of Gastrointestinal Surgery, Kanagawa Cancer Center, 1-1-2 Nakao, Asahi-ku, Yokohama 241-0815, Japan e-mail: yoshikawat@kcch.jp

\section{Introduction}

5-Fluorouracil (5-FU)-based chemotherapy is widely used for unresectable advanced or recurrent gastric cancer and has a survival benefit in comparison to the best supportive care [1]. Two phase III studies to evaluate chemotherapy regimens for gastric cancer were recently reported from Japan [2, 3]. The JCOG9912 trial compared 5-FU to S-1 alone or cisplatin (CDDP) plus irinotecan (CPT-11), and found $\mathrm{S}-1$ alone to be comparable to 5-FU alone, but CDDP plus CPT-11 therapy failed to demonstrate superiority to 5-FU alone in overall survival (OS; 11.4 vs. 12.3 vs. 10.8 months). The SPIRITS trial compared the efficacy of S-1 plus CDDP to that of S- 1 alone, and found that S- 1 plus CDDP showed a significantly longer overall survival (OS; 13 vs. 11 months; $P=0.037$ ). These trials included patients with recurrent gastric cancer who did not receive adjuvant chemotherapy or those who received an oral fluoropyrimidine other than S-1. However, prior to these studies, no drugs had been confirmed to be effective as adjuvant chemotherapy after curative surgery.

The ACTS-GC trial first demonstrated that S-1 was effective as adjuvant chemotherapy for Japanese patients who underwent curative gastrectomy for locally advanced gastric cancer and were diagnosed as pathological stage II or III [4]. Therefore, adjuvant S-1 chemotherapy has been established as the standard therapy for stage II or III gastric cancer in Japan. However, about 30\% of the patients still develop recurrence after a curative resection followed by adjuvant S-1. The survival of patients who experience recurrence after adjuvant $\mathrm{S}-1$ has not been fully clarified. It is unclear whether these patients should be treated as candidates for first-line chemotherapy.

The present study investigated the survival, and the factors that could predict the survival, in gastric cancer 
patients who experienced recurrence after adjuvant chemotherapy with $\mathrm{S}-1$ and thereafter received palliative chemotherapy.

\section{Patients and methods}

Patients

Patients were selected from the database of the Kanagawa Cancer Center, Department of Gastrointestinal Surgery, Yokohama, Japan, according to the following criteria: (1) histologically proven gastric adenocarcinoma, (2) patients who underwent a curative surgical resection for gastric cancer as a primary treatment between June 2002 and December 2009, (3) stage II, IIIA, or IIIB determined pathologically according to the guidelines of the Japanese Gastric Cancer Association[5], (4) patients who received adjuvant S-1 chemotherapy after surgery for more than 4 weeks at a starting dose of $80 \mathrm{mg} / \mathrm{m}^{2},(5)$ recurrence was confirmed by computed tomography (CT), magnetic resonance imaging (MRI), barium enema, laparoscopy, or bone scintigraphy.

\section{Evaluation and statistical analyses}

The overall survival (OS) was calculated from the date of the imaging study that confirmed the recurrence to the date of any cause of death or last follow-up. Unpaired Student's $t$-test or the $\chi^{2}$ method was used to compare two groups. Survival curves were calculated using the Kaplan-Meier method and compared by the log-rank test. Cox's proportional hazard model was used to perform univariate and stepwise multivariate survival analyses. A $P$ value of $<0.05$ was defined to be statistically significant, and the data were expressed as medians \pm ranges.

An SPSS software package (v11.0 J Win; SPSS, Chicago, IL, USA) was used for all statistical analyses.

\section{Results}

A total of 233 patients underwent surgical resection and were pathologically diagnosed as stage II, IIIa, or IIIb. Among them, 92 patients received adjuvant chemotherapy with S-1. Thirty-four patients were eligible for the present study. The median follow-up was 21.5 months ( range from 4.3 to 57.2 months). The median OS was 7.3 months (95\% confidence interval [CI], 5-9.6 months). Twenty-six patients received palliative chemotherapy after recurrence, while 8 did not, due to renal dysfunction in 2 , liver dysfunction in 1, mechanical intestinal obstruction in 1, and patient's refusal in 4 . The median OS was 8.5 months $(95 \%$

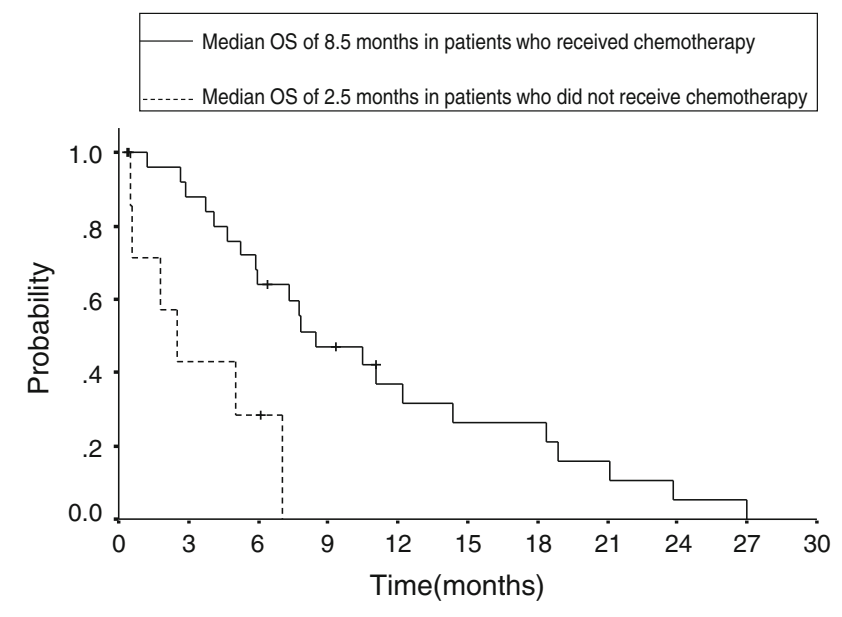

Fig. 1 Kaplan-Meier curves for overall survival $(O S)$ showed a significant difference between patients who received chemotherapy (solid line) and those who did not receive chemotherapy (broken line; $P=0.0022)$

CI, 4.4-12.5 months) in the patients who received chemotherapy and 2.5 months (95\% CI, 0.7-4.3 months) in those who did not, and the difference was statistically significant $(P=0.0022$; Fig. 1$)$.

The backgrounds of the 26 patients who received chemotherapy are shown in Table 1 . None of the 26 patients received any other therapies, such as a surgical resection or radiological treatment, in addition to chemotherapy during the clinical course.

Prognosticators in these patients were analyzed by univariate and multivariate analyses. The median duration of adjuvant S-1 administration was 6.2 months, with a range from 1 to 19.9 months. Six patients stop S-1 for $\leqq 3$ months due to toxicity. The treatment was withdrawn in 8 of the remaining patients before 6 months, due to recurrence in 5 , toxicity in 2 , and for other reasons in 1 . The treatment was withdrawn in 6 of the remaining patients before 9 months, due to recurrence in 3 and for other reasons in 3 . As a result, 8 patients discontinued S1 due to recurrence and 12 patients discontinued S1 due to toxicity or other reasons. The chemotherapy regimens after recurrence were individually selected by the patient's physician. One patient received S-1, 21 received taxane-containing regimens [taxane group (i.e., paclitaxel and docetaxel)], and 4 received irinotecan plus cisplatin (CPT-11 group).

A univariate analysis of factors affecting OS demonstrated that histological type was the only significant factor (Table 2). The OS of the differentiated type was significantly better than that of the undifferentiated type $(P=0.009$; Fig. 2). The multivariate analysis revealed that histological type remained the only independent significant prognosticator (Table 3). However, the duration of 
Table 1 Background of patients who received chemotherapy

\begin{tabular}{|c|c|}
\hline Age (years) & $58.6 \pm 11.6$ \\
\hline \multicolumn{2}{|l|}{ Gender } \\
\hline Male & 16 \\
\hline Female & 10 \\
\hline \multicolumn{2}{|l|}{ PS (ECOG) at recurrence } \\
\hline 0 & 18 \\
\hline 1 & 8 \\
\hline \multicolumn{2}{|l|}{ Histological type } \\
\hline Differentiated & 9 \\
\hline Undifferentiated & 17 \\
\hline \multicolumn{2}{|l|}{ Pathological stage } \\
\hline Stage II & 4 \\
\hline Stage III A & 9 \\
\hline Stage III B & 13 \\
\hline \multicolumn{2}{|l|}{ Site of recurrence } \\
\hline Peritoneum & 14 \\
\hline Liver & 5 \\
\hline Lymph node & 5 \\
\hline Other & 2 \\
\hline Disease-free interval, months median (range) & $13.1(3.9-38.9$ \\
\hline \multicolumn{2}{|l|}{ Duration of adjuvant $S-1$} \\
\hline$<3$ Months & 6 \\
\hline$\geqq 3$ Months & 20 \\
\hline \multicolumn{2}{|l|}{ Treatment-free interval (since last S-1) } \\
\hline$<6$ Months & 13 \\
\hline$\geqq 6$ Months & 13 \\
\hline \multicolumn{2}{|l|}{ Disease-free interval (since surgery) } \\
\hline$<12$ Months & 12 \\
\hline$\geqq 12$ Months & 14 \\
\hline \multicolumn{2}{|l|}{ First-line chemotherapy after recurrence } \\
\hline Taxane group & 21 \\
\hline CPT-11 group & 4 \\
\hline S-1 & 1 \\
\hline \multicolumn{2}{|l|}{ Second-line chemotherapy after recurrence } \\
\hline Taxane group & 5 \\
\hline CPT-11 group & 6 \\
\hline
\end{tabular}

$P S$ performance status, ECOG Eastern Cooperative Oncology Group, $C P T$ irinotecan

chemotherapy tended to be significant according to the univariate analysis, but not based on the multivariate analysis.

Figure 3 shows details of the regimens of the first- and second-line chemotherapy in 9 patients with the differentiated type and 17 with the undifferentiated type. Most patients received taxane-containing regimens as the firstline chemotherapy. The proportion of patients who received both taxanes and irinotecan was higher in those with the differentiated type (6 of 9 patients, 66.7\%) than in those with the undifferentiated type (3 of 17 patients,
Table 2 Univariate Cox proportional hazards analysis of clinicopathologic factors

\begin{tabular}{|c|c|c|c|}
\hline Factor (category) & $\begin{array}{l}\text { No. of } \mathrm{OR} \\
\text { patients }\end{array}$ & $95 \% \mathrm{CI}$ & $P$ value \\
\hline
\end{tabular}

Age

0.164

$<65$ Years

1.000

$\geqq 65$ Years

$9 \quad 2.204 \quad 0.724-6.716$

PS (ECOG)

0

0.136

1

1.000

Histological type

Differentiated

Undifferentiated

Duration of adjuvant S-1

$18 \quad 1.000$

$\begin{array}{lll}8 & 2.315 & 0.768-6.975\end{array}$

$<3$ Months

$\geqq 3$ Months

Treatment-free interval

(since last S-1)

$<6$ Months

$\geqq 6$ Months

$13 \quad 2.026 \quad 0.755-5.433$

Recurrence-free interval (since surgery)

$\begin{array}{llll}<12 \text { Months } & 12 & 1.000 & \\ \geqq 12 \text { Months } & 14 & 1.737 \quad 0.689-4.383\end{array}$

Site of recurrence

Peritoneum

Other

$14 \quad 1.000$

$12 \quad 0.688 \quad 0.282-1.682$

First-line chemotherapy after recurrence

$\begin{array}{lrrr}\text { S-1 } & 1 & 1.000 & \\ \text { CPT-11 group } & 4 & 0.590 & 0.076-4.545 \\ \text { Taxane group } & 21 & 0.427 & 0.097-1.886\end{array}$

0.483

$O R$ odds ratio, $C I$ confidence interval, $P S$ performance status, $E C O G$ Eastern Cooperative Oncology Group

$17.6 \%)$, and the difference was statistically significant $(P=0.012)$.

\section{Discussion}

Only Shitara et al. [6] retrospectively examined the efficacy and survival of the treatment in patients who developed recurrence after adjuvant S-1 chemotherapy. The response rate to S-1-containing chemotherapy was $0 \%$. They recommended other chemotherapeutic regimens in this setting. Most patients in the present study received taxane-containing regimens. Only 1 patient received palliative S-1 after recurrence. Despite the use of taxanes in most patients, the median OS of the 26 patients who received chemotherapy after recurrence was only 8.5 months, which did not reach the level expected for 
first-line chemotherapy for gastric cancer. Shitara [6] reported the median OS was only 9.1 months with S-1containing chemotherapy and 10.1 months with a non-S-1-

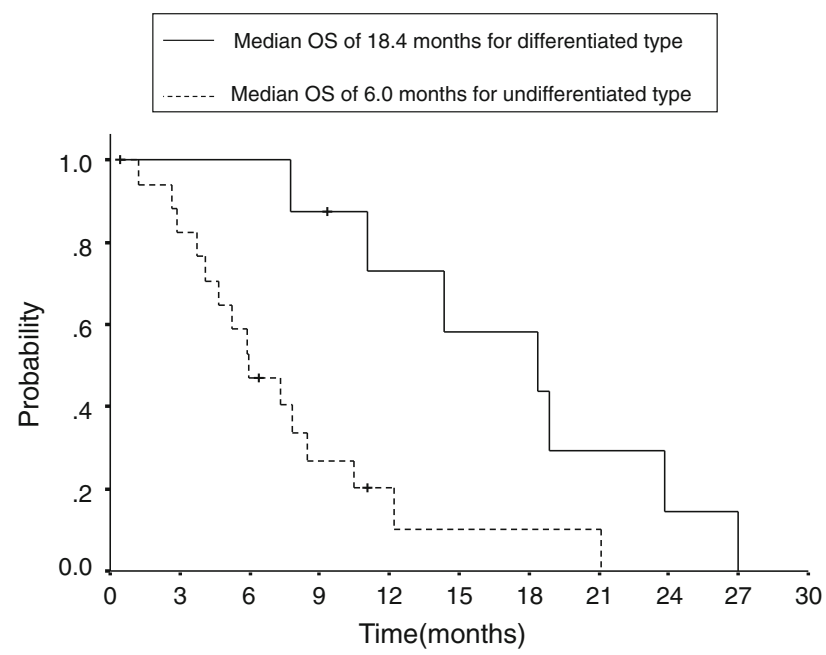

Fig. 2 Kaplan-Meier curves for overall survival $(O S)$ showed a significant difference between patients with the differentiated type (solid line) and those with the undifferentiated type (broken line; $P=0.009)$

Table 3 Stepwise multivariate Cox proportional hazards analysis of clinicopathologic factors

\begin{tabular}{llcll}
\hline $\begin{array}{l}\text { Factor } \\
\text { (category) }\end{array}$ & $\begin{array}{l}\text { No. of } \\
\text { patients }\end{array}$ & $P$ value & $\begin{array}{l}\text { Hazard } \\
\text { ratio }\end{array}$ & 95\% CI \\
\hline $\begin{array}{l}\text { Histological type } \\
\text { (Differentiated versus undifferentiated) }\end{array}$ & 9 and 17 & 0.009 & 4.117 & $1.420-11.931$ \\
\hline
\end{tabular}

containing regimen. These results suggest that, in patients who have recurrence after adjuvant S-1 chemotherapy, the disease may have to be treated as refractory to $\mathrm{S}-1$.

Histological type is not known as a prognosticator in first-line chemotherapy for gastric cancer. The present study is the first to demonstrate that histological type was the only significant prognosticator by univariate and multivariate analyses in patients with recurrence after adjuvant S-1. On the other hand, some authors have reported the significance of the histological type in the survival of preoperative patients or in sensitivity to chemotherapy. Adachi et al. [7] evaluated 504 preoperative patients with gastric cancer that was classified as welldifferentiated and poorly differentiated types. They found the 5-year survival rate to be higher in patients with welldifferentiated gastric carcinoma than that in patients with poorly differentiated gastric carcinoma. Futatsuki et al. [8] reported a late phase II study of CPT-11 in advanced gastric cancer that found that the response rate was higher in patients with differentiated types than those with undifferentiated types (30.0 vs. 14.3\%). On the other hand, Mai et al. [9] reported a late phase II study of docetaxel in advanced gastric cancer and found that the response rate was similar in patients with differentiatedtype cancer and those with undifferentiated type (20.0 vs. $26.3 \%$ ). In addition, two phase II studies of paclitaxel in advanced gastric cancer showed that the response rates for diffuse- and intestinal-types were 29 and $17 \%$, and 36 and $24 \%$, respectively $[10,11]$. These reports may suggest that the histological type is important for chemosensitivity. which determines survival especially in S-1-refractory tumors. Patients with a differentiated type may have a greater chance of responding to both taxanes and CPT-11

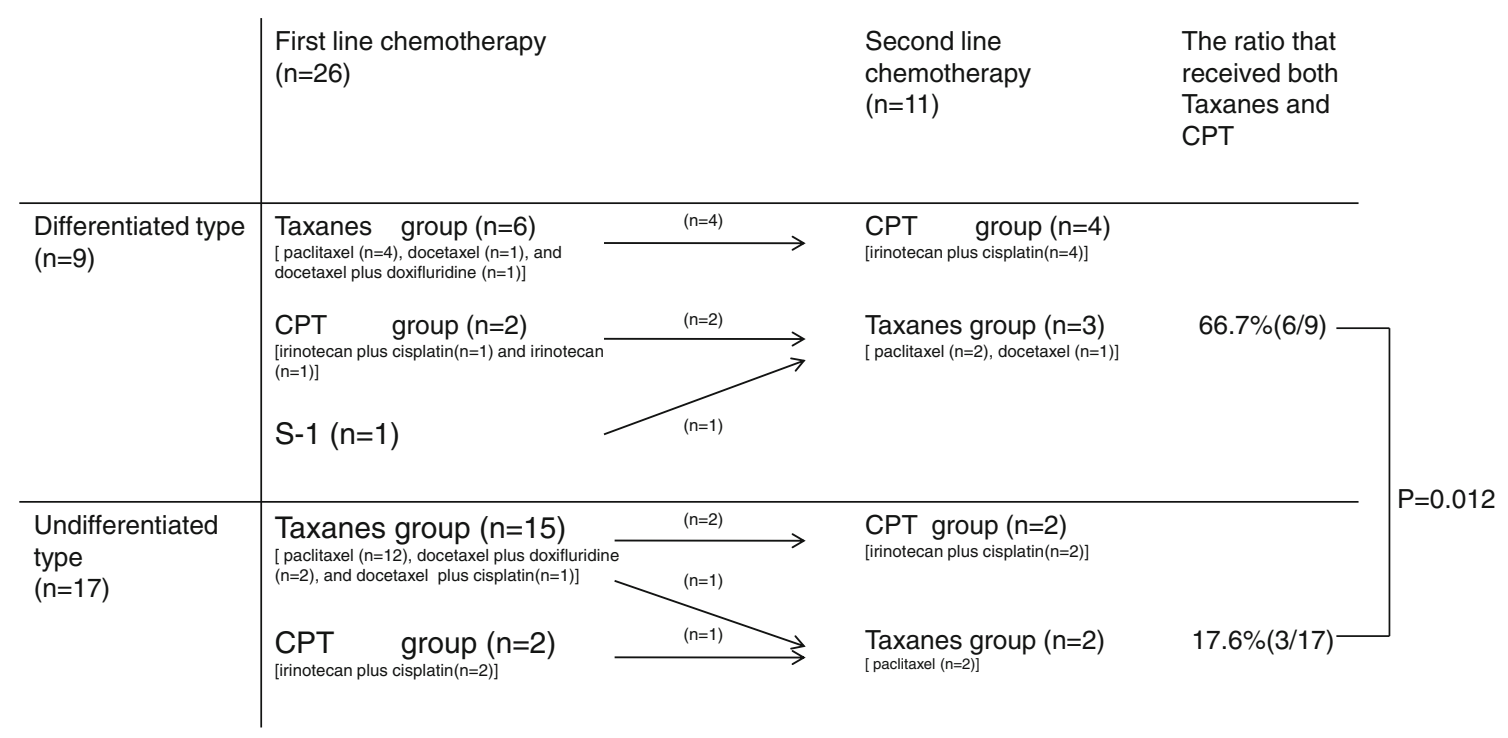

Fig. 3 Details of the first line- and second-line chemotherapy regimens in 9 patients with the differentiated type and 17 with the undifferentiated type 
in comparison to those with an undifferentiated type, which would thereby contribute to the survival.

The present study found that $66.7 \%$ of patients with the differentiated type received both taxanes and CPT-11, in comparison to $17.6 \%$ of those with the undifferentiated type. This difference may have affected the difference in the survival between the two types. In particular, only 2 patients received CPT-11 as second-line chemotherapy among 15 patients with the undifferentiated type who had received taxanes as first-line chemotherapy, which decreased the rate of the entry into the second-line chemotherapy and may have shortened the survival. However, the undifferentiated type has more chance of responding to taxanes than CPT-11, as mentioned above. It is unclear whether or not the survival of the undifferentiated type is improved by selecting CPT-11 as the first-line chemotherapy.

Of note, the duration of the S-1 adjuvant chemotherapy did not have a significant prognostic impact in our study. Although a group who received S-1 for 3 months or longer tended to have a lower risk of recurrence compared with a group who received $\mathrm{S}-1$ for $<3$ months, the difference did not reach statistical significance. Moreover, multivariate analysis identified the histological type as the only independent significant prognostic factor. Nevertheless, the duration of S-1 chemotherapy could, in theory, be relevant, and there is a possibility that the small number of patients analyzed might have adversely affected our results. The reasons for discontinuation of $\mathrm{S}-1$ should also be taken into consideration when discussing the prognostic impact of the treatment duration. Again, given the small sample size, it was not practical at this time to analyze survival by further subdividing the patients into those who discontinued treatment due to toxicity and those whose treatment was terminated due to recurrence. In addition to the issue of sample size, the retrospective nature of the study and diversity of the drugs used after S-1 failure are weaknesses that need to be borne in mind when interpreting results from the present study.

In summary, the present study revealed that survival after failing the standard adjuvant chemotherapy did not reach the expected 12 months as observed in recent phase III trials for untreated advanced/metastatic gastric cancer. Undifferentiated phenotype was a significant indicator of poor prognosis in these patients.
Acknowledgments This work was supported, in part, by Kanagawa Health Foundation.

Conflict of interest None declared.

\section{References}

1. Murad AM, Santiago FF, Petroianu A, Rocha PR, Rodrigues MA, Rausch M. Modified therapy with 5-fluorouracil, doxorubicin, and methotrexate in advanced gastric cancer. Cancer. 1993;72:37-41.

2. Boku N, Yamamoto S, Fukuda H, Shirao K, Doi T, Sawaki A, et al. Fluorouracil versus combination of irinotecan plus cisplatin versus S-1 in metastatic gastric cancer: a randomised phase 3 study. Lancet Oncol. 2009;10:1027-8.

3. Koizumi W, Narahara H, Hara T, Takagane A, Akiya T, Takagi $\mathrm{M}$, et al. S-1 plus cisplatin versus S-1 alone for first-line treatment of advanced gastric cancer (SPIRITS trial): a phase III trial. Lancet Oncol. 2008;9:215-21.

4. Sakuramoto S, Sasako M, Yamaguchi T, Kinoshita T, Fujii M, Nashimoto A, et al. Adjuvant chemotherapy for gastric cancer with S-1, an oral fluoropyrimidine. N Engl J Med. 2007;357: 1810-20.

5. Japanese Gastric Cancer Association. Japanese classification of gastric carcinoma 2nd English edition. Gastric Cancer. 1998;1:10-24.

6. Shitara K, Muro K, Ura T, Takahari D, Yokota T, Sawaki A, et al. Chemotherapy for gastric cancer that recurs after adjuvant chemotherapy with S-1. Jpn J Clin Oncol. 2008;38:786-9.

7. Adachi Y, Yasuda K, Inomata M, Sato K, Shiraishi N, Kitano S. Pathology and prognosis of gastric carcinoma: well versus poorly differentiated type. Cancer. 2000;89:1418-24.

8. Futatsuki K, Wakui A, Nakao I, Sakata Y, Kambe M, Shimada Y. Late phase II study of irinotecan hydrochloride (CPT-11) in advanced gastric cancer. Gan To Kagaku Ryoho. 1994;21: 1033-8.

9. Mai M, Sakata Y, Kanamaru R, Kurihara M, Suminaga M, Ota J, et al. A late phase II clinical study of RP56976 (docetaxel) in patients with advanced or recurrent gastric cancer: a cooperative study group trial (group B). Gan To Kagaku Ryoho. 1999;26: 487-96.

10. Yamada Y, Shirao K, Ohtsu A, Boku N, Hyodo I, Saitoh H, et al. Phase II trial of paclitaxel by three-hour infusion for advanced gastric cancer with short premedication for prophylaxis against paclitaxel-associated hypersensitivity reactions. Ann Oncol. 2001;12:1133-7.

11. Yamaguchi K, Tada M, Horikoshi N, Otani T, Takiuchi H, Saitoh S, et al. Phase II study of paclitaxel with 3-h infusion in patients with advanced gastric cancer. Gastric Cancer. 2002;5:90-5. 